QUARTERLY OF APPLIED MATHEMATICS

VOLUME LXIV, NUMBER 3

SEPTEMBER 2006, PAGES 401-412

S $0033-569 X(06) 01037-2$

Article electronically published on August 17, 2006

\title{
PARAMETRIZATION OF THE TWO AND THREE-DIMENSIONAL MOTION OF A VISCOUS INCOMPRESSIBLE LIQUID
}

\author{
BY
}

\author{
K. B. RANGER
}

Department of Mathematics, University of Toronto, Toronto, Ontario M5S 3 G3 Canada

\begin{abstract}
A method is described for parametrizing the velocity components and space coordinates in terms of parametric functions and time for the two and threedimensional motion of a viscous incompressible liquid. The two-dimensional motion contains four functions and the three-dimensional motion contains six functions satisfying minimal requirements.
\end{abstract}

Introduction. It is desirable in theoretical fluid dynamics to express physical quantities such as fluid velocity, pressure, and vorticity as explicit known functions of the space coordinates and time. This approach leads to a more tractable way for the solution of boundary value and initial value problems. In many situations, this is successful especially for linearized versions of the momentum equations. However, in cases where the full nonlinearity of the quasi-linear autonomous governing equations is taken into account the problem is often insurmountable and the only methodology available is through asymptotic methods, C.F.D. techniques or a combination of both.

Much of theoretical fluid mechanics is understood in terms of these powerful, welldeveloped strategies. There does exist the possibility that, for global flows, these methods cannot be guaranteed to elucidate the intrinsic and mysterious properties of the nonlinear convective-acceleration term contained in the flow equations.

The theory of differential equations derived from standard texts cites examples of differential equations where the solutions can be exhibited in a straightforward manner by parametrization of the dependent and independent variables. The problem of expressing the dependent variable as an explicit function of the independent variables may be complicated or intractable. For example, in the case of an ordinary differential equation, consider

$$
x=p e^{p}, p=\frac{d y}{d x} .
$$

Received May 12, 2004

2000 Mathematics Subject Classification. Primary 76-xx, 76Dxx.

(C)2006 Brown University Reverts to public domain 28 years from publication 
By differentiation with respect to $y$ and inverting the role of $\frac{d p}{d y}$, followed by integration, it is found that the general solution is described by

$$
x=p e^{p}, y=\left(p^{2}-p+1\right) e^{p}+\text { constant } .
$$

The singular solution of the extended Clairaut equation

$$
y=p x+f(p), p=\frac{d y}{d x}
$$

is found by direct differentiation and is expressed by

$$
x=-f^{\prime}(p), y=f(p)-p f^{\prime}(p) .
$$

In the case of partial differential equations, the analogue of the Clairaut equations is given by

$$
z=p x+q y+f(p, q), p=z_{x}, q=z_{y},
$$

where $f(p, q)$ is a general function of $p, q$ with $\left(f_{p p} f_{q q}-f_{p q}^{2}\right) \neq 0$. Then there is a solution in parametric form found by differentiation and provided by

$$
\begin{aligned}
& x=-f_{p}(p, q), y=-f_{q}(p, q), \\
& z=f(p, q)-p f_{p}(p, q)-q f_{q}(p, q) .
\end{aligned}
$$

Even though it may not be possible to eliminate the parametric functions in all three cases, the solutions are known in a computational or numerical sense and can be graphed in a straightforward manner.

For the differential equations of fluid dynamics, the Lagrange-Legendre contact transformation and the hodograph mapping ([1], 2]) are both methods of parametrization and useful for describing the steady two-dimensional motion of a compressible inviscid fluid.

It is noted that, even though the parametrization of dependent and independent variables may be expressed in terms of elementary functions, the eliminant of the parameters even when it can be carried out may be excessively cumbersome in terms of standard known special functions. This appears to be the case with nonlinear equations and serves as a motivation for the methodology of 2 and 3 dimensional viscous motion.

The two-dimensional motion of a viscous incompressible fluid. The fluid velocity components $u, v$ can be expressed in terms of a stream function $\psi=\psi(x, y, t)$ by

$$
u=-\psi_{y}, v=\psi_{x}
$$

and the momentum equations can be written as

$$
\begin{aligned}
-\psi_{y t}-\psi_{x} w & =-H_{x}^{\prime}-\nu w_{y} \\
\psi_{x t}-\psi_{y} w & =-H_{y}^{\prime}+\nu w_{x}
\end{aligned}
$$

where

$$
w=\psi_{x x}+\psi_{y y}, H^{\prime}=p / \rho_{0}+\frac{1}{2}\left(\psi_{x}^{2}+\psi_{y}^{2}\right)
$$


$w$ is the vorticity and $H^{\prime}$ is the Bernoulli function or total head of pressure. For the present purpose, it is appropriate to write $H^{\prime}=H+f(x)+g(y)$, where $f, g$ are differentiable functions such that $f^{\prime}(x), g^{\prime}(y)$ have unique inverses in the fluid region. In this case, equations (2), (3) can be written as

$$
\begin{aligned}
& f^{\prime}(x)=w \psi_{x}-H_{x}-\nu w_{y}+\psi_{y t}, \\
& g^{\prime}(y)=w \psi_{y}-H_{y}+\nu w_{x}-\psi_{x t},
\end{aligned}
$$

or equivalently,

$$
\begin{aligned}
x & =f^{\prime-1}\left(w \psi_{x}-H_{x}-\nu w_{y}+\psi_{y t}\right), \\
y & =g^{\prime-1}\left(w \psi_{y}-H_{y}+\nu w_{x}-\psi_{x t}\right) .
\end{aligned}
$$

In this article, the method of approach is to consider two differentiable functions $F=$ $F(\psi, H, t), G=G(\psi, H, t)$ such that

$$
x=F(\psi, H, t), y=G(\psi, H, t)
$$

and the mapping of $\psi, H$ into $x, y$ is a diffeomorphism or a one-to-one continuously differentiable mapping requiring that

$$
F_{\psi} G_{H}-F_{H} G_{\psi} \neq 0
$$

in the fluid region. Now partial differentiation of equations (9) produces the equations

$$
\begin{gathered}
\psi_{x}=A=\frac{G_{H}}{F_{\psi} G_{H}-F_{H} G_{\psi}}, H_{x}=B=\frac{-G_{\psi}}{F_{\psi} G_{H}-F_{H} G_{\psi}}, \\
\psi_{y}=C=\frac{-F_{H}}{F_{\psi} G_{H}-F_{H} G_{\psi}}, H_{y}=D=\frac{F_{\psi}}{F_{\psi} G_{H}-F_{H} G_{\psi}}, \\
\psi_{t}=J=\frac{F_{H} G_{t}-G_{H} F_{t}}{F_{\psi} G_{H}-F_{H} G_{\psi}}, H_{t}=k=\frac{G_{\psi} F_{t}-F_{\psi} G_{t}}{F_{\psi} G_{H}-F_{H} G_{\psi}},
\end{gathered}
$$

where

$$
\begin{gathered}
A=A(\psi, H, t), \quad B=B(\psi, H, t), C=C(\psi, H, t), \\
D=D(\psi, H, t), \quad J=J(\psi, H, t), K=K(\psi, H, t) .
\end{gathered}
$$

With further differentiation, it is possible to calculate $w, w_{x}, w_{y}$ in terms of $A, B, C, D$, and their derivatives. These are expressed by

$$
\begin{gathered}
w=A A_{\psi}+B A_{H}+C C_{\psi}+D C_{H}=E, \\
w_{x}=A E_{\psi}+B E_{H}, w_{y}=c E_{\psi}+D E_{H},
\end{gathered}
$$

where $E=E(\psi, H, t)$ and, explicitly,

$$
\begin{gathered}
E_{\psi}=A A_{\psi \psi}+A_{\psi}^{2}+B A_{\psi H}+A_{H} B_{\psi}+A C_{\psi \psi}+C_{\psi} A_{\psi}+D C_{\psi H}+C_{H} D_{\psi}, \\
E_{H}=A A_{\psi H}+A_{\psi} A_{H}+B A_{H H}+A_{H} B_{H}+A C_{\psi H}+C_{\psi} A_{H}+D C_{H H}+C_{H} D_{H} .
\end{gathered}
$$

In addition,

$$
\psi_{x t}=A J_{\psi}+B J_{H}, \psi_{y t}=C K_{\psi}+D K_{H}
$$

and at this point $x, y$ can be expressed in terms of $\psi, H, t$, in the forms

$$
x=f^{\prime-1}(P), y=g^{\prime-1}(Q),
$$


where

$$
\begin{aligned}
& P=A\left[A A_{\psi}+B A_{H}+C C_{\psi}+D C_{H}\right]-B+C J_{\psi}+D J_{H}-\nu\left(C E_{\psi}+D E_{H}\right), \\
& Q=C\left[A A_{\psi}+B A_{H}+C C_{\psi}+D C_{H}\right]-D-A J_{\psi}+\nu\left(A J_{\psi}+B J_{H}\right) .
\end{aligned}
$$

In these representations $A, B, C, D, J, K$ are functions of $\psi, H, t$ so that in turn $P=$ $P(\psi, H t), Q=Q(\psi, H, t)$ are also functions of $\psi, H, t$, where $F$ and $G$ are subject to the restriction (10). It follows that the components of fluid velocity $(u, v)$ and $H_{x}^{\prime}, H_{y}^{\prime}$ together with coordinates $(x, y)$ can be parametrized in terms of $\psi, H$. Explicitly these quantities can be written as

$$
\begin{gathered}
u=\bar{h} \psi_{y}=-C(\psi, H, t), v=\psi_{x}=A(\psi, H, t), \\
H_{x}^{\prime}=f^{\prime}(x)+B(\psi, H, t), H_{y}^{\prime}=g^{\prime}(y)+D(\psi, H, t), \\
x=f^{\prime-1}\left\{A\left[A A_{\psi}+B A_{H}+C C \psi+D C_{H}\right]-B+C J_{\psi}+D J_{H}-\nu\left(C E_{\psi}+D E_{H}\right)\right\}, \\
y=g^{\prime-1}\left\{C\left[A A_{\psi}+B A_{H}+C C_{\psi}+D C_{H}\right]-D-A J_{\psi}-B J_{H}+\nu\left(A J_{\psi}+B J_{H}\right)\right\}
\end{gathered}
$$

which are subject to the restrictions

$$
F_{\psi} G_{H}-F_{H} G_{\psi} \neq 0, P_{\psi} Q_{H}-P_{H} Q_{\psi} \neq 0
$$

in the fluid region. However, justification is required since there are two representations for $x, y$ expressed by

$$
\begin{aligned}
& x=F(\psi, H, t)=f^{\prime-1}(P(\psi, H, t)), \\
& y=G(\psi, H, t)=g^{\prime-1}(Q(\psi, H, t)),
\end{aligned}
$$

and by elimination of $x, y$ it is found that

$$
f^{\prime}(F)=P(\psi, H, t), g^{\prime}(G)=Q(\psi, H, t) .
$$

In this system $F, G$ are the dependent variables and $\psi, H, t$ are the independent variables. In view of (26), it is possible to invert the roles of dependent and independent variables and recast equations (29) in which $\psi, H$ are the dependent variables and $F, G, t$ are the independent variables. With $x=F, y=G$, the result of these transformations is to reproduce the momentum equations in the form

$$
\begin{aligned}
-\psi_{G t}-w \psi_{F} & =-H_{F}^{\prime}-\nu w_{G}, \\
\psi_{F t}-w \psi_{G} & =-H_{G}^{\prime}+\nu W_{F},
\end{aligned}
$$

where

$$
w=\psi_{F F}+\psi_{G G}, H^{\prime}=H+f(F)+f(G) .
$$

There are exact solutions, see [4, given by

$$
\begin{aligned}
\psi & =\frac{\left(1+\nu s^{2}\right)}{s} F+e^{s G-t}, \\
H & =\frac{1}{2} s^{2} e^{2(G s-t)}-f(F)-g(G),
\end{aligned}
$$


where $s<0$ is a constant. Now inspection of the conditions (26) shows that they are essentially equivalent to the one condition

$$
X=\psi_{F} H_{G}-\psi_{G} H_{F} \neq 0
$$

in the fluid and for equations (33) it is found that

$$
X=\frac{\left(1+\nu s^{2}\right)}{s}\left[s^{3} e^{2(G s-t)}-g^{\prime}(G)\right]+s e^{G s-t} f^{\prime}(F),
$$

which can be satisfied by general functions $g^{\prime}(G)>0, f^{\prime}(F)<0$, so that $X$ is always positive.

At this point, consistency has been achieved and it is only necessary to replace $\psi, H$ by (33) in the expressions for $u, v, x, y$. In this way the velocity components and planar variables are expressed in terms of four functions $F, G, f, g$. The results may be summarized by the set of equations

$$
x=f^{\prime^{-1}}(P), y=g^{\prime^{-1}}(Q),
$$

where $P=P(\psi, H, t)$ and $Q=Q(\psi, H, t)$ are expressed by equations (20), (21). The velocity components $u, v$ are described by

$$
u=-C, v=A,
$$

where $A=A(\psi, H, t), C=C(\psi, H, t)$ are defined by (11) and (12). Finally $\psi, H$ are expressed by (33), that is,

$$
\begin{gathered}
\psi=\frac{\left(1+\nu s^{2}\right) F}{s}+e^{s G-t}, \\
H=\frac{1}{2} s^{2} e^{2\left(G_{s-t}\right)}-f(F)-g(G) .
\end{gathered}
$$

Since $A, C, P, Q$ contain the functions $F, G, f, g$ the physical quantities are all expressed in terms of these functions, which represent a parametric representation of the flow under general conditions.

These equations demonstrate the generality of the method of parametrization for describing the physical quantities as solutions of a nonlinear dynamical system such as the Navier-Stokes equations. The prospect of eliminating the parameters by special choice of the functions $F, G$ to determine explicit expressions for the physical quantities in terms of the space variables and time is not clear at the present time. However, the form of representation for the physical quantities and space variables does lend itself to numerical computation. For assigned choices of the functions $F, G$ it is possible to calculate the velocity components numerically from corresponding numerical values of the parameters.

A more detailed description for the determination of a more general type of parametric solution derived from the flow equations is presented in 6]. It appears that there is more than one method for carrying out this task. Basically, it depends on the fact that when a parametric solution is known from a differential equation, then the parameters can always be modified to accommodate other solutions.

A possible application of the results described here is for the generalization of global motion forced by specific flow singularities. This will be be pursued here as it seems that the main results are of more significance at least from a theoretical viewpoint. 
It is noted that the functions $F, G$ are not required to satisfy partial differential equations and are only subject to the minimal restrictions imposed by (40). There are no parallel results for linear partial differential equations as the functions $F, G$ would be required to satisfy differential equations.

One advantage of the method described here is for the direct extension to threedimensional motion. This is demonstrated in the next section.

The three-dimensional motion of a viscous fluid. The Navier-Stokes equations for the motion of a viscous incompressible liquid can be expressed in the form

$$
\begin{gathered}
q_{t}+[\underline{\Omega} \times \underline{q}]=-\nabla H+\nu \nabla^{2} \underline{q}, \\
\operatorname{div} \underline{q}=(\underline{\nabla} \cdot \underline{q})=0,
\end{gathered}
$$

where $\underline{q}=\underline{q}(x, y, z, t)$ is the fluid velocity, $\underline{\Omega}=[\underline{\nabla} \times \underline{q}]$ is the vorticity vector, $H=$ $p / \rho_{0}+\frac{\overline{1}}{2}|\underline{q}|^{2}$ is the Bernoulli function and $\nu$ is the kinematic viscosity assumed to be finite and nonzero. If $\underline{q}=u \hat{\imath}+v \hat{\jmath}+w \hat{k}$, where $(u, v, w)$ are functions of the space coordinates and time $t$, it is convenient for the present purposes to write $H=C+f(x)+g(y)+h(z)$, where the components of equation (41) are expressed by

$$
\begin{aligned}
& f^{\prime}(x)=\nu \nabla^{2} u-u_{t}-(\hat{\imath} \cdot[\underline{\Omega} \times \underline{q}])-C_{x}=P, \\
& g^{\prime}(y)=\nu \nabla^{2} v-v_{t}-(\hat{\jmath} \cdot[\underline{\Omega} \times \underline{q}])-C_{y}=Q, \\
& h^{\prime}(z)=\nu \nabla^{2} w-w_{t}-(\hat{k} \cdot[\underline{\Omega} \times \underline{q}])-C_{z}=R .
\end{aligned}
$$

It is assumed that $f^{\prime}(x), g^{\prime}(y), h^{\prime}(z)$ have unique inverse functions in the fluid. Now under general circumstances, the equation of continuity is satisfied by writing

$$
\underline{q}=\operatorname{curl}(A \hat{\imath}+B \hat{\jmath})=-B_{z} \hat{\imath}+A_{z} \hat{\jmath}+\left(B_{x}-A_{y}\right) \hat{k},
$$

where $A, B$ are independent general functions of $x, y, z, t$. In this case,

$$
u=-B_{z}, v=A_{z}, w=B_{x}-A_{y} .
$$

The vorticity vector $\underline{\Omega}$ is given by

$$
\underline{\Omega}=\operatorname{curl}^{2}(A \hat{\imath}+B \hat{\jmath})=\left(B_{x y}-A_{y y}-A_{z z}\right) \hat{\imath}+\left(A_{x y}-B_{x x}-B_{z z}\right) \hat{\jmath}+\left(A_{x z}+B_{y z}\right) \hat{k}
$$

and the nonlinear convective acceleration vector is expressed by

$$
\begin{aligned}
{[\underline{\Omega} \times \underline{q}] } & =\left\{\left(B_{x}-A_{y}\right)\left(A_{x y}-B_{x x}-B_{z z}\right)-A_{z}\left(A_{x z}+B_{y z}\right)\right\} \hat{\imath} \\
& +\left\{\left(A_{y}-B_{x}\right)\left(B_{x y}-A_{y y}-A_{z z}-B_{z}\left(A_{x q}+B_{y z}\right)\right\} \hat{\jmath}\right. \\
& +\left\{A_{z}\left(B_{x y}-A_{y y}-A_{z z}\right)+B_{z}\left(A_{x y}-B_{x x}-B_{z z}\right)\right\} \hat{k} .
\end{aligned}
$$


In this case $x, y, z$ can be expressed in terms of the functions $A, B, C$ with their partial derivatives and are represented by

$$
\begin{aligned}
x=f^{\prime-1}(P) & =f^{\prime-1}\left\{-\nu \nabla^{2} B_{z}+B_{z t}-C_{x}+A_{z}\left(A_{x z}+B_{y z}\right)\right. \\
& \left.-\left(B_{x}-A_{y}\right)\left(A_{x y}-B_{x x}-B_{z z}\right)\right\} \\
y=g^{\prime-1}(Q) & =g^{\prime-1}\left\{\nu \nabla^{2} A_{z}-A_{z t}-C_{y}+B_{z}\left(A x z+B_{y z}\right)\right. \\
& \left.\quad\left(A_{y}-B_{x}\right)\left(B_{x y}-A_{y y}-A_{z z}\right)\right\} \\
z=h^{\prime-1}(R) & =h^{\prime-1}\left\{\nu \nabla^{2} B_{x}-\nu \nabla^{2} A_{y}-B_{x t}+A_{y t}\right. \\
& \left.\quad-C_{z}-A_{z}\left(B_{x y}-A_{y y}-A_{z z}\right)-B_{z}\left(A_{x y}-B_{x x}-B_{z z}\right)\right\} .
\end{aligned}
$$

The fact that $u, v, w, x, y, z$ are expressed only in terms of $A, B, C$ partial derivatives lends itself to the opportunity of parametrizing these quantities in terms of $A, B, C, t$ containing three arbitrary functions subject to minimal restrictions. It will be shown that $P, Q, R$ can be expressed as functions of $A, B, C, t$, and this is demonstrated by the following analysis that

$$
F=F(A, B, C, t), F^{\prime}=F^{\prime}(A, B, C, t), F^{\prime \prime}=F^{\prime \prime}(A, B, C, t)
$$

be three differentiable and independent functions satisfying the equations

$$
x=F(A, B, C, t), y=F^{\prime}(A, B, C, t), z=F^{\prime \prime}(A, B, C, t)
$$

and subject to the requirement

$$
\Gamma=\left|\begin{array}{ccc}
F_{A} & F_{B} & F_{0} \\
F_{A}^{\prime} & F_{B}^{\prime} & F_{C}^{\prime} \\
F_{A}^{\prime \prime} & F_{B}^{\prime \prime} & F_{C}^{\prime \prime}
\end{array}\right| \begin{gathered}
=F_{A}\left(F_{B}^{\prime} F_{C}^{\prime \prime}-F_{C}^{\prime} F_{B}^{\prime \prime}\right)+F_{B}\left(F_{C}^{\prime} F_{A}^{\prime \prime}-F_{A}^{\prime} F_{C}^{\prime \prime}\right) \\
+F_{0}\left(F_{A}^{\prime} F_{B}^{\prime \prime}-F_{B}^{\prime} F_{A}^{\prime \prime}\right) \neq 0 .
\end{gathered}
$$

In this case the mapping $x, y, z$ into $A, B, C$ is a diffeomorphism and by partial differentiation it is found that

$$
\begin{gathered}
F_{A} A_{x}=F_{B} B_{x}+F_{C} C_{x}=1, \\
F_{A}^{\prime} A_{x}=F_{B}^{\prime} B_{x}+F_{C}^{\prime} C_{x}=0, \\
F_{A}^{\prime \prime} A_{x}=F_{B}^{\prime \prime} B_{x}+F_{C}^{\prime \prime} C_{x}=0, \\
F_{A} A_{y}+F_{B} B_{y}+F_{C} C_{y}=0, \\
F_{A}^{\prime} A_{y}+F_{B}^{\prime} B_{y}+F_{C}^{\prime} C_{y}=1, \\
F_{A}^{\prime \prime} A_{y}+F_{B}^{\prime \prime} B_{y}+F_{C}^{\prime \prime} C_{y}=0, \\
F_{A} A_{z}+F_{B} B_{z}+F_{C} C_{z}=0, \\
F_{A}^{\prime} A_{z}+F_{B}^{\prime} B_{z}+F_{C}^{\prime} C_{z}=0, \\
F_{A}^{\prime \prime} A_{z}+F_{B}^{\prime \prime} B_{z}+F_{C}^{\prime \prime} C_{z}=1, \\
F_{A}^{\prime \prime} A_{t}+F_{B} B_{t}+F_{C} C_{t}=-F_{t}, \\
F_{A}^{\prime} A_{t}+F_{B}^{\prime} B_{t}+F_{C}^{\prime} C_{t}=-F_{t}^{\prime}, \\
F_{A}^{\prime \prime} A_{t}+F_{B}^{\prime \prime} B_{t}+F_{C}^{\prime \prime} C_{t}=-F_{t}^{\prime \prime} .
\end{gathered}
$$


In order to derive explicit expressions for the first derivatives of $A, B, C$, it is useful to recall Cramer's Rule [3] for a system of $3 \times 3$ linear equations in the form

$$
\begin{gathered}
\alpha R_{1}+\beta R_{2}+\gamma R_{3}=S, \\
\alpha^{\prime} R_{1}+\beta^{\prime} R_{2}+\gamma^{\prime} R_{3}=S^{\prime}, \\
\alpha^{\prime \prime} R_{1}+\beta^{\prime \prime} R_{2}+\gamma^{\prime \prime} R_{3}=S^{\prime \prime} .
\end{gathered}
$$

The solutions for $R_{1}, R_{2}, R_{3}$, in terms of the other variables, are provided by

$$
\begin{aligned}
& R_{1}=\frac{1}{\Gamma^{\prime}}\left\{S\left(\beta^{\prime} \gamma^{\prime \prime}-\gamma^{\prime} \beta^{\prime \prime}\right)+S^{\prime}\left(\gamma \beta^{\prime \prime}-\beta \gamma^{\prime \prime}\right)+S^{\prime \prime}\left(\beta \gamma^{\prime}-\gamma \beta^{\prime}\right)\right\}, \\
& R_{2}=\frac{1}{\Gamma^{\prime}}\left\{S\left(\alpha^{\prime \prime} \gamma^{\prime}-\alpha^{\prime} \gamma^{\prime \prime}\right)+S^{\prime}\left(\alpha \gamma^{\prime \prime}-\gamma \alpha^{\prime}\right)+S^{\prime \prime}\left(\gamma \alpha^{\prime}-\alpha \gamma^{\prime}\right)\right\}, \\
& R_{3}=\frac{1}{\Gamma^{\prime}}\left\{S\left(\alpha^{\prime} \beta^{\prime \prime}-\beta^{\prime} \alpha^{\prime \prime}\right)+S^{\prime}\left(\beta \alpha^{\prime \prime}-\alpha \beta^{\prime \prime}\right)+S^{\prime \prime}\left(\alpha \beta^{\prime}-\beta \alpha^{\prime}\right)\right\},
\end{aligned}
$$

where

$$
\Gamma^{\prime}=\left|\begin{array}{ccc}
\alpha & \beta & \gamma \\
\alpha^{\prime} & \beta^{\prime} & \gamma^{\prime} \\
\alpha^{\prime \prime} & \beta^{\prime \prime} & \gamma^{\prime \prime}
\end{array}\right|=\alpha\left(\beta^{\prime} \alpha^{\prime \prime}-\alpha^{\prime} \beta^{\prime \prime}\right)+\beta\left(\partial^{\prime} \alpha^{\prime \prime}-\alpha^{\prime} \partial^{\prime \prime}\right)
$$

With appropriate designations for $\alpha, \beta, \gamma, \alpha^{\prime}, \beta^{\prime}, \gamma^{\prime}, \alpha^{\prime \prime}, \beta^{\prime \prime}, \gamma^{\prime \prime}, S, S^{\prime}, S^{\prime \prime}$ it is found that

$$
\begin{gathered}
A_{x}=a=\frac{1}{\Gamma}\left(F_{B}^{\prime} F_{C}^{\prime \prime}-F_{C}^{\prime} F_{B}^{\prime \prime}\right), B_{x}=a^{\prime}=\frac{1}{\Gamma}\left(F_{C}^{\prime} F_{A}^{\prime \prime}-F_{A}^{\prime} F_{C}^{\prime \prime}\right), \\
C_{x}=a^{\prime \prime}=\frac{1}{\Gamma}\left(F_{A}^{\prime} F_{B}^{\prime \prime}-F_{B}^{\prime} F_{A}^{\prime \prime}\right) .
\end{gathered}
$$

In the same way it is found that

$$
\begin{gathered}
A_{y}=b=\frac{1}{\Gamma}\left(F_{C} F_{B}^{\prime \prime}-F_{B} F_{C}^{\prime \prime}\right), B_{y}=b^{\prime}=\frac{1}{\Gamma}\left(F_{A} F_{C}^{\prime \prime}-F_{B} F_{A}^{\prime \prime}\right), \\
C_{y}=b^{\prime \prime}=\frac{1}{\Gamma}\left(F_{B} F_{A}^{\prime \prime}-F_{A} F_{B}^{\prime \prime}\right), \\
A_{z}=c=\frac{1}{\Gamma}\left(F_{B} F_{C}^{\prime}-F_{C} F_{B}^{\prime}\right), B_{z}=c^{\prime}=\frac{1}{\Gamma}\left(F_{C} F_{A}^{\prime}-F_{A} F_{C}^{\prime}\right), \\
C_{z}=\frac{1}{\Gamma}\left(F_{A} F_{B}^{\prime}-F_{B} F_{A}^{\prime}\right)=c^{\prime \prime}
\end{gathered}
$$

and finally

$$
\begin{aligned}
& A_{t}=d=-\frac{1}{\Gamma}\left\{F_{t}\left(F_{B}^{\prime} F_{C}^{\prime \prime}-F_{C}^{\prime} F_{B}^{\prime \prime}\right)+F_{t}^{\prime}\left(F_{C} F_{B}^{\prime \prime}-F_{B} F_{C}^{\prime \prime}\right)+F_{t}^{\prime \prime}\left(F_{B} F_{C}^{\prime}-F_{C} F_{B}^{\prime}\right)\right\}, \\
& B_{t}=d^{\prime}=-\frac{1}{\Gamma}\left\{F_{t}\left(F_{C}^{\prime} F_{A}^{\prime \prime}-F_{A}^{\prime} F_{C}^{\prime \prime}\right)+F_{t}^{\prime}\left(F_{A} F_{C}^{\prime \prime}-F_{C} F_{A}^{\prime \prime}\right)+F_{t}^{\prime \prime}\left(F_{C} F_{A}^{\prime}-F_{A} F_{C}^{\prime}\right)\right\}, \\
& C_{t}=d^{\prime \prime}=-\frac{1}{\Gamma}\left\{F_{t}\left(F_{A}^{\prime} F_{B}^{\prime \prime}-F_{B}^{\prime} F_{A}^{\prime \prime}\right)+F_{t}^{\prime}\left(F_{B} F_{A}^{\prime \prime}-F_{A} F_{B}^{\prime \prime}\right)+F^{\prime \prime}\left(F_{A} F_{B}^{\prime}-F_{B} F_{A}^{\prime}\right)\right\}
\end{aligned}
$$

It can be verified directly by partial differentiation that the first derivatives of $A, B, C$ do in fact satisfy the consistency or integrability requirements that $A_{x y}=A_{y x}$, etc. However, it is a cumbersome calculation to carry out this exercise directly, and it is preferable to return to the original set of equations defined through (14)-(16), for example, and differentiate (14) with respect to $y$ and $z,(15)$ with respect to $x$ and $z$, and (16) with respect to $x$ and $y$. In this way the desired results can be achieved with less algebra. 
The conditions $A_{x t}=A_{t x}$, etc., are obtained in a similar manner. In these derivations and calculations the functions $F, F^{\prime}, F^{\prime \prime}$ are basically unrestricted apart from continuity and differentiability requirements together with the constraint imposed by (54).

In order to complete the analysis and express the space coordinates in terms of functions $A, B, C, t$ it is necessary from (14), (15), (16), etc. to calculate the second derivatives of $A, B$ and some third derivatives of these functions. This is achieved in a concise form by using equations (74)-(82) and expressing the required derivatives in terms of the derivatives of $a, b, c, d$, etc.

In this way it is found that

$$
\begin{gathered}
A_{x x}=a a_{A}+a^{\prime} a_{B}+a^{\prime \prime} a_{C}, A_{x y}=b a_{A}+b^{\prime} a_{B}+b^{\prime \prime} a_{C}, \\
A_{x z}=c a_{A}+c^{\prime} a_{B}+c^{\prime \prime} a_{C}, A_{x t}=d a_{A}+d^{\prime} a_{B}+d^{\prime \prime} a_{C}, \\
A_{y y}=b b_{A}+b^{\prime} b_{B}+b^{\prime \prime} b_{C}, A_{y z}=c b_{A}+c^{\prime} b_{B}+c^{\prime \prime} b_{C}, \\
A_{y t}=d b_{A}+d^{\prime} b_{B}+b_{C} d^{\prime \prime}, A_{z z}=c c_{A}+c^{\prime} c_{B}+c_{C}, \\
A_{z t}=d c_{A}+d^{\prime} c_{B}+d^{\prime \prime} c_{C}, A_{z z}=c c_{A}+c^{\prime} c_{B}+c_{C}, \\
B_{x x}=a a_{A}^{\prime}+a^{\prime} a_{B}^{\prime}+a^{\prime \prime} a_{C}^{\prime}, A_{x y}=b a_{A}^{\prime}+b^{\prime} a_{B}^{\prime}+b^{\prime \prime} a_{C}^{\prime}, \\
B_{y y}=b b_{A}^{\prime}+b^{\prime} b_{B}^{\prime}+b^{\prime \prime} b_{C}^{\prime}, B_{x z}=c a_{A}^{\prime}+c^{\prime} a_{B}^{\prime}+c^{\prime \prime} a_{C}^{\prime}, \\
B_{x t}=d a_{A}^{\prime}+\alpha^{\prime} a_{B}^{\prime}+\alpha^{\prime \prime} a_{C}^{\prime}, B_{y z}=c b_{A}^{\prime}+c^{\prime} b_{B}^{\prime}+c^{\prime \prime} b_{C}^{\prime}, \\
B_{y t}=d b_{A}^{\prime}+d^{\prime} b_{B}^{\prime}+d^{\prime \prime} b_{C}^{\prime}, B_{z z}=c c_{A}^{\prime}+c^{\prime} c_{B}^{\prime}+c^{\prime \prime} c_{C}^{\prime}, \\
B_{z t}=d c_{A}^{\prime}+d^{\prime} c_{B}^{\prime}+\alpha^{\prime \prime} c_{C}^{\prime} .
\end{gathered}
$$

For the third derivatives only $\frac{\partial}{\partial y} \nabla^{2} A, \frac{\partial}{\partial z} \nabla^{2} A, \frac{\partial}{\partial x} \nabla^{2} B, \frac{\partial}{\partial z} \nabla^{2} B$ are required and to this end

$$
\begin{aligned}
& \nabla^{2} A=A^{*}=a a_{A}+a^{\prime} a_{B}+a^{\prime \prime} a_{C}+b b_{A}+b^{\prime} b_{B}+b^{\prime \prime} b_{C}+c c_{A}+c^{\prime} c_{B}+c^{\prime \prime} c_{C}^{\prime}, \\
& \nabla^{2} B=B^{*}=a a_{A}^{\prime}+a^{\prime} a_{B}^{\prime}+a^{\prime \prime} a_{C}^{\prime}+b b_{A}^{\prime}+b^{\prime} b_{B}^{\prime}+b^{\prime \prime} b_{C}^{\prime}+c c_{A}^{\prime}+c^{\prime} c_{B}^{\prime}+c^{\prime \prime} c_{C}^{\prime},
\end{aligned}
$$

where $A^{*}, B^{*}$ are functions of $A, B, C, t$. It follows that

$$
\begin{aligned}
& \frac{\partial}{\partial y} \nabla^{2} A=b A_{A}^{*}+b^{\prime} A_{B}^{*}+b^{\prime \prime} A_{C}^{*}, \\
& \frac{\partial}{\partial z} \nabla^{2} A=c A_{A}^{*}+b^{\prime} A_{B}^{*}+c^{\prime \prime} A_{C}^{*}, \\
& \frac{\partial}{\partial x} \nabla^{2} B=a B_{A}^{*}+a^{\prime} B_{B}^{*}+a^{\prime \prime} B_{C}^{*}, \\
& \frac{\partial}{\partial z} \nabla^{2} B=c B_{A}^{*}+c^{\prime} B_{B}^{*}+c^{\prime \prime} B_{C}^{*} .
\end{aligned}
$$

At this point all of the required derivatives have been calculated as functions of $A, B, C, t$ containing $F, F^{\prime}, F^{\prime \prime}$, which are so far only subject to the restriction (57). In this case it follows that the functions $P, Q, R$ can also be expressed as functions of 
$A, B, C, t$, and the space coordinates can be written as

$$
\begin{gathered}
x=f^{\prime-1}(P(A, B, C, t)), y=g^{-1}(Q(A, B, C, t)), \\
z=h^{\prime-1}(R(A, B, C, t)) .
\end{gathered}
$$

Also the fluid velocity components are given by

$$
u=-c^{\prime}, v=c, w=a^{\prime}-b,
$$

all of which are functions of $A, B, C, t$. In a similar way,

$$
H_{x}=a^{\prime \prime}+f^{\prime}(x), H_{y}=b^{\prime \prime}+g^{\prime}(y), H_{z}=c^{\prime \prime}+h^{\prime}(z)
$$

are functions of $A, B, C, t$ and since $H_{t}=C_{t}=d^{\prime \prime}$ it follows formally that

$$
\begin{aligned}
H & =\int H_{x} d t+H_{y} d y+H_{z} d z+H_{t} d t \\
& =\int\left(a^{\prime \prime}+f^{\prime}(x) d x+\left(b^{\prime \prime}+g^{\prime}(y)\right) d y+\left(c^{\prime \prime}+h^{\prime}(z)\right) d z+d^{\prime \prime} d t,\right.
\end{aligned}
$$

which is also a function of $A, B, C, t$.

It follows that the physical quantities and space variables $x, y, z$ are parametrized in terms of $A, B, C, t$, and the functions $F, F^{\prime}, F^{\prime \prime}$ are subject to the conditions (57) and

$$
\left|\begin{array}{ccc}
P_{A} & P_{B} & P_{C} \\
Q_{A} & Q_{B} & Q_{C} \\
R_{A} & R_{B} & R_{C}
\end{array}\right|=\begin{gathered}
P_{A}\left(Q_{B} R_{C}-Q_{C} R_{B}\right)+P_{B}\left(Q_{C} R_{A}-Q_{A} R_{C}\right) \\
+P_{C}\left(Q_{A} R_{B}-Q_{B} R_{A}\right) \neq 0
\end{gathered}
$$

in the fluid so that the mapping is a diffeomorphism.

As in the two-dimensional case the method of derivation requires justification in view of the results

$$
\begin{gathered}
f^{\prime}(x)=P, x=F, g^{\prime}(y)=Q, y=F^{\prime}, \\
h^{\prime}(z)=R, z=F^{\prime \prime}
\end{gathered}
$$

or by elimination of $x, y, z$,

$$
f^{\prime}(F)=P, g^{\prime}\left(F^{\prime}\right)=Q, h^{\prime}\left(F^{\prime \prime}\right)=R .
$$

The equations represent a system of nonlinear partial differential equations in which $F, F^{\prime}, F^{\prime \prime}$ are the dependent variables and $A, B, C, t$ are the independent variables. If the condition (59) is satisfied, then the roles of dependent and independent variables can be inverted and the recast system contains $A, B, C, t$ as dependent variables and $F, F^{\prime}, F^{\prime \prime}, t$ as independent variables. The resulting system of equations are the flow equations in the form

$$
\underline{q}+\underline{\Omega} \times \underline{q}=-\underline{\Omega} H+\nu \nabla^{2} \underline{q},
$$

where

$$
\begin{gathered}
\underline{q}=[\underline{\nabla} \times(A \hat{\imath}+B \hat{\jmath})], \underline{\nabla}=\hat{\imath} \frac{\partial}{\partial F}+\hat{\jmath} \frac{\partial}{\partial F^{\prime}}+\hat{k} \frac{\partial}{\partial F^{\prime \prime}}, \\
H=C+f(F)+g\left(F^{\prime}\right)+h\left(F^{\prime \prime}\right), \\
\underline{\Omega}=[\underline{\nabla} \times[\underline{\nabla} \times(A \hat{\imath}+B \hat{\jmath})]], \nabla^{2}=\frac{\partial^{2}}{\partial F^{2}}+\frac{\partial^{2}}{\partial F^{\prime 2}}+\frac{\partial^{2}}{\partial F^{\prime \prime 2}} .
\end{gathered}
$$


Also as in the two-dimensional case, the two conditions (54), (108) reduce to a single condition defined by

$$
\left|\begin{array}{ccc}
A_{F} & B_{F} & C_{F} \\
A_{F}^{\prime} & B_{F}^{\prime} & C_{F}^{\prime} \\
A_{F}^{\prime \prime} & B_{F}^{\prime \prime} & C_{F}^{\prime \prime}
\end{array}\right|=A_{F}\left(B_{F^{\prime}} C_{F^{\prime \prime}}-C_{F^{\prime}} B_{F^{\prime \prime}}\right)+B_{F}\left(C_{F^{\prime}} A_{F^{\prime \prime}}-A_{F^{\prime}} C_{F^{\prime \prime}}\right)
$$

in the fluid region.

Again suitable forms for $A$ and $B$ from which $C$ can be determined are given by

$$
\begin{gathered}
A=a_{0} F^{\prime \prime}+a_{1} F+a_{2} e^{s F^{\prime}-t}, \\
B=b_{0} F^{\prime \prime}+b_{1} F+b_{2} e^{s F^{\prime}-t} .
\end{gathered}
$$

Then $C$ is found to be expressed by

$$
C=\frac{1}{2} a_{2}^{2} s^{2} e^{2 s F^{\prime}-2 t}-a_{2} s b_{1} e^{s F^{\prime}-t}-f(F)-g\left(F^{\prime}\right)-h\left(F^{\prime \prime}\right)
$$

where

$$
a_{0}=\left(1+\nu s^{2}\right) / s .
$$

Again at this point consistency has been achieved and it only remains to substitute the expressions for $A, B, C$ into the corresponding equations representing the velocity components $(u, v, w)$ and the space coordinates $x, y, z$. This will not be carried out directly but the ultimate results are that $u, v, x, y, z$ are all expressible in terms of the six functions $F, F^{\prime}, F^{\prime \prime}, f, g, h$ subject to the minimal restriction that the required mapping is a diffeomorphism.

Conclusion. A parametrization has been constructed for the full Navier-Stokes equations describing the theoretical motion of a viscous incompressible liquid, with three parametric functions, containing six general functions satisfying basically minimal requirements. Even with this generality, there are many solutions excluded by this analysis such as those of similarity type. The immediate application of these results is to the motion for global flows forced by singularities, and this has been demonstrated in [5] for the two-dimensional case using a different method of derivation. It is not clear at this time that the flow representation can be applied to either boundary value or initial value problems. It seems that, in view of the generality of the results, it is worth putting on record as a method which circumvents the difficulty of the convective-acceleration term in the flow equations, and also there are no parallel results for linear partial differential equations with the same generality.

\section{REFERENCES}

[1] W.F. Ames, Nonlinear Partial Differential Equations in Engineering, Vol. II, Academic Press, 1972, p. 38. MR0473442 (57:13108)

[2] R. Von Mises, Mathematical Theory of Compressible Fluid Flow, Applied Mathematics and Mechanics, vol. 3, Academic Press, New York, 1958, p. 85. MR0094996 (20:1504)

[3] G. Birkhoff and S. Mac Lane, A Survey of Modern Algebra, Macmillan Company of New York, 1941, p. 306. MR0005093 (3:99h)

[4] R. Berker, Intégration des équations du mouvement d'un fluide visqueux incompressible, Handbuch der Physik VIII/2, Springer, Berline, 1963, pp. 1-386. MR0161513 (28:4717) 
[5] K. B. Ranger, Fluid velocity fields derived from vorticity singularities, Quart. Appl. Math. 62 (2004), 671-685. MR2104268 (2005f:76035)

[6] K. B. Ranger, Parametric solutions for differential equations, submitted for publication 2006. 